Trauma Berufskrankh 2006 · 8[Suppl 3]: S293-S295

DOI 10.1007/s10039-005-1069-8

Online publiziert: 1. Dezember 2005

(c) Springer Medizin Verlag 2005

\section{Rolle und Funktion des MDK im Verhältnis zu seinen Trägern}

In einem „Positionspapier zu Rolle und Funktion des Medizinischen Dienstes im Verhältnis zu seinen Trägern" des Vorstandes des Medizinischen Dienstes der Spitzenverbände der Krankenkassen (MDS) [4], der „Bundesorganisation der Landes$\mathrm{MDK}^{\text {“, sind die wesentlichsten Aussagen }}$ hierzu präzisiert:

- Der MDK ist vom Gesetzgeber als Kassenarten übergreifende Arbeitsgemeinschaft definiert.

- Der MDK ist Teil der Gesetzlichen Krankenversicherung (GKV) und der Sozialen Pflegeversicherung (SPV). Er ist den Aufgaben und Zielen der GKV und SPV verpflichtet und erfüllt seine Aufgaben in Übereinstimmung mit ihnen.

- Der MDK hat im Verhältnis zur GKV und SPV - ebenso wie die Krankenund die Pflegekassen zu ihren Versicherten - die Funktion eines Dienstleisters.

- Der MDK ist in seiner medizinischfachlichen Bewertung unabhängig und frei von Anbieterinteressen.

- Der MDK berücksichtigt als Teil der GKV und SPV deren Gesamtinteressen bei seiner Aufgabenwahrnehmung.

Daraus ergeben sich für den MDK die im Folgenden aufgeführten Schwerpunktaufgaben.

R.-G. Matthesius

Medizinischer Dienst der Krankenversicherung Berlin-Brandenburg e. V., Potsdam

\title{
Rolle des beratenden Arztes aus der Sicht des MDK
}

\section{Schwerpunktaufgaben des MDK}

In seiner Dienstleistungsfunktion arbeitet der MDK primär auftragsbezogen.

Das SGB V weist ihm 2 wesentliche Kernaufgaben zu:

1. Die umfassende Unterstützung der Gesetzlichen Kranken- und Pflegekassen bei Leistungsentscheidungen und bei der Sicherstellung einer bundeseinheitlichen Leistungsgewährung (grundsatz- und/oder einzelfallbezogene Aufträge)

Eine rationale Leistungssteuerung durch die Gesetzlichen Kranken- und Pflegekassen ist in weiten Bereichen ohne sozialmedizinischen, fachmedizinischen und pflegerischen Sachverstand nicht möglich. In ihrer Entwicklung vom „payer zum player“ benötigen sie ständig hochwertige, ggf. auch juristisch belastbare, sozial- und fachmedizinische Expertisen, um die täglich im Rahmen ihrer Prüfungen grundsätzlich und einzelfallbezogen anfallenden Fragen zur Notwendigkeit sowie zu Art, Umfang und Wirtschaftlichkeit der Leistungsgewährung sachlich richtig beantworten $\mathrm{zu}$ können.

Unabhängig von seinem Wohnort und unabhängig von seiner gewählten gesetzlichen Kranken- bzw. Pflegekasse ist in Deutschland für jeden Versicherten bei Erfüllung der Anspruchsvoraussetzungen die gleiche Leistung zu garantieren. Der MDK ist an der Sicherung dieser bedeutenden sozialpolitischen Errungenschaft Deutschlands maßgeblich beteiligt.
Die gutachtlichen Empfehlungen des MDK werden von seinen Auftraggebern in der Regel auch als Grundlage für die grundsätzlich und im Einzelfall zu treffenden leistungsrechtlichen Entscheidungen genutzt.

2. Die Unterstützung der Gesetzlichen Kranken- und Sozialen Pflegekassen bei der strukturellen Gestaltung des gesundheitlichen Versorgungssystems (struktur-, versorgungs- und systembezogene Aufträge)

In den letzten Jahren wird der MDK zunehmend stärker in die Strukturpolitik der gesetzlichen Kranken- und Pflegekassen einbezogen. Als aktuelles Beispiel hierfür ist die Nutzung des fachgutachtlichen Sachverstands beim MDK Berlin-Brandenburg e. V. zur Vorbereitung von Vertragsverhandlungen mit Leistungsanbietern zu nennen.

Darüber hinaus wird der gewachsene Sachverstand des MDK Berlin-Brandenburg e. V. von seinen Auftraggebern zunehmend auch in die Vorbereitung und Durchführung von Beratungen zu Strukturfragen integriert. Die Einbeziehung des MDK in diesen Aufgabenkomplex war zum Zeitpunkt dessen Gründung vor 16 Jahren noch eine Vision.

Die mit diesen Kernaufgaben verbundenen Erwartungen seiner Träger erfüllt der MDK Berlin-Brandenburg e. V. im Rahmen seiner Tätigkeit und hält die hierfür notwendigen Ressourcen vor. 
Trauma Berufskrankh 2006 -8[Suppl 3]:S293-S295

c) Springer Medizin Verlag 2005

R.-G. Matthesius

\section{Rolle des beratenden Arztes aus der Sicht des MDK}

\section{Zusammenfassung}

Die Bedeutung des MDK ist im Zuge der zunehmenden Veränderungen des Gesundheitsversorgungssystems deutlich gewachsen. Die Träger des Medizinischen Dienstes der Krankenversicherung sowie die Leistungserbringer und deren Interessenvertretungen erwarten vom MDK eine auftragsbezogen zeitnahe und qualifizierte Arbeit. Der MDK Berlin-Brandenburg e. V. stellt sich im Konsens mit seinen Trägern den gewachsenen Anforderungen an seine sozialmedizinische und fachmedizinische Kompetenz. Bei der Klärung möglicher Erstattungsansprüche zwischen Krankenkassen und Unfallversiche- rungsträgern ist er für seine Auftraggeber sozialmedizinisch und fachmedizinisch beratend, begutachtend und prüfend tätig. Bei ihm werden diese Aufträge zentral bearbeitet. Zur Sicherstellung einer effizienten, fachkompetenten und einheitlichen Abarbeitung sind erfahrene und sozialmedizinisch/sozialrechtlich geschulte Fachgutachter im Einsatz.

\section{Schlüsselwörter}

Föderalistische Organisation · Sozial- und fachmedizinische Kompetenz $\cdot$ Krankenversicherung · Unfallversicherung · Restitution

\section{The role of the consultant - as seen by the MDK}

\begin{abstract}
Because of the increasingly frequent changes in the German healthcare system, the "Medizinischer Dienst der Krankenversicherung" (medical review and advisory board for health insurance - MDK) has grown in importance. The body responsible for the MDK, and also those responsible to provide the achievements of health care and their representations of interest, expect up-to-date and professional work from the MDK. The MDK Berlin-Brandenburg, the local MDK for the region of the German capital, is facing up to the higher demands on its competence in terms of social medicine and medical specialisms. If there are problems between the firms provid-
\end{abstract}

ing required health insurance and the statutory accident insurance firms, the MDK is there to provide consultation, expert assessments, and tests. Such duties are handled by a central office of the MDK Berlin-Brandenburg. To ensure efficient, competent and uniform treatment experienced assessors with training in social medicine and social rights legislation are available.

\section{Keywords}

Federal organization - Competence in social medicine and medical specialisms . Compulsory health insurance - Statutory accident insurance $\cdot$ Restitution

\section{Zusammenarbeit zwischen KV, MDK und UVT}

Im Folgenden wird auf die Frage eingegangen, welche Rolle der MDK in den Arbeitsbeziehungen zwischen den Gesetzlichen Krankenkassen und den Trägern der Unfallversicherung (UVT) spielt. Im Wesentlichen geht es dabei um Fragen der Leistungs- und Zuständigkeitsabgrenzung bei Arbeitsunfällen und Berufserkrankungen zwischen den Krankenkassen und den UVT.

Die gesetzlichen Grundlagen hierfür bieten der $\$_{91}$ SGB X (Erstattung von Aufwendungen) und die $\$ \$ 102 \mathrm{ff}$. SGB X (Erstattungsansprüche der Leistungserbringer untereinander). Es besteht somit die Möglichkeit bzw. die Notwendigkeit, die erbrachten Leistungsaufwendungen beim jeweils anderen Leistungsträger im Rahmen eines Erstattungsanspruchs zu regressieren.

Die Krankenkassen unterstützen ihre Versicherten bei Notwendigkeit auch in Fragen zu Arbeitsunfällen und bei der Klärung des Verdachts auf das Vorliegen einer Berufserkrankung. So weit möglich, führen die hierfür eingesetzten Mitarbeiter der Krankenkassen die notwendigen Klärungen im Dialog mit den UVT selbstständig durch. Zur Prüfung der Frage, ob und in welchem Umfang ein Erstattungsanspruch zu realisieren oder abzuwenden ist, bedarf es allerdings oftmals der Klärung medizinischer Sachverhalte. Die Unterstützungsleistung des MDK für die Krankenkassen ergibt sich aus $\$ 275$ SGB V (Begutachtung und Beratung) sowie den „Richtlinien über die $\mathrm{Zu}$ sammenarbeit der Krankenkassen mit den Medizinischen Diensten der Krankenversicherung" vom August 1990 (s. dort unter Punkt 3.4.2 "Zusammenhangsfragen") [3].

Die im Rahmen dieser Auftragserteilungen an den MDK Berlin-Brandenburg e. V. am häufigsten gestellten Fragen der Krankenkassen beziehen sich auf folgende Schwerpunkte (Reihenfolge nicht nach Häufigkeit):

- fraglicher Zusammenhang zwischen Unfall (Ereignis) und Erkrankung

- Unfall (Ereignis) aus innerer Ursache

- Unfall (Ereignis) aus Gelegenheitsursache 
- fraglicher Zusammenhang (Kausalität) zwischen Unfallfolgen und Verordnung, Arbeitsunfähigkeit, stationärer Behandlung u. a. m.

- unfallbedingte Verschlimmerung eines vorbestehenden Leidens

- Abgrenzung von Unfall- und Nichtunfallfolgen (z. B. Fragen zur Dauer der unfallbedingten Arbeitsunfähigkeit, der Dauer unfallbedingter Krankenhausbehandlung)

- fraglicher Zusammenhang zwischen dem Unfall(Ereignis) und der vorliegenden Pflegestufe

- Einschätzung des Zukunftsrisikos

- Verdacht auf Berufserkrankung

Der MDK Berlin-Brandenburg e. V. ist in diesem Arbeitsbereich für die Krankenkassen sozialmedizinisch beratend, begutachtend und prüfend tätig. Seitens der auftraggebenden Krankenkasse werden dem MDK Berlin-Brandenburg e. V. in der Regel die zur Beantwortung der Fragestellung benötigten Unterlagen zur Verfügung gestellt.

Voraussetzung für diese anspruchsvollen Tätigkeiten und zur effizienten Auftragsbearbeitung ist für die einbezogenen MDK-Gutachter neben der medizinischen Fachkompetenz auch die Kenntnis der sozialmedizinischen und sozialrechtlichen Begrifflichkeiten aus dem Recht der gesetzlichen Unfallversicherung. Zur Gewährleistung einer effizienten, fachkompetenten und einheitlichen Bearbeitung werden diese Aufträge beim MDK Berlin-Brandenburg e. V. in einem speziellen Arbeitsbereich „Medizinischjuristische Fragen“ (Med-Jur. Fragen) von erfahrenen und geschulten Fachgutachtern zentral bearbeitet [5]. Bei Notwendigkeit sind im Einzelfall eine übergreifende Unterstützung innerhalb der MDKGemeinschaft oder die Hinzuziehung von externem Sachverstand möglich.

\section{Korrespondierender Autor}

\section{Dr. R.-G. Matthesius}

Medizinischer Dienst der Krankenversicherung Berlin-Brandenburg e. V. Konrad-Wolf-Allee 1-3, TH III, 14480 Potsdam rolf-gerd.matthesius@mdk-bb.de

Interessenkonflikt. Es besteht kein Interessenkonflikt. Der korrespondierende Autor versichert, dass keine Verbindungen mit einer Firma, deren Produkt in dem Artikel genannt ist, oder einer Firma, die ein Konkurrenzprodukt vertreibt, bestehen. Die Präsentation des Themas ist unabhängig und die Darstellung der Inhalte produktneutral.

\section{Literatur}

1. Matthesius RG (2004) Die Entwicklung des MDK Berlin-Brandenburg e. V. Gemeinsames Symposium MDK Berlin-Brandenburg e. V. und MDS e. V.: Entwicklung und Perspektiven des Medizinischen Dienstes der Krankenversicherung. 16.09.2004, Berlin

2. Matthesius RG (2005) Entwicklung und Perspektiven des MDK. Hauptstadtkongress 2005 Medizin und Gesundheit: Der MDK im Spannungsfeld zwischen Kontrolle und Dienstleistung. Berlin, 15.17.06.2005

3. Medizinischer Dienst der Spitzenverbände der Krankenkassen e. V. (1990) Richtlinien über die Zusammenarbeit der Krankenkassen mit den Medizinischen Diensten der Krankenversicherung nach §213 SGB V am 27.08.1990 als Richtlinie nach §282 Satz 3 SGB V beschlossen. Medizinischer Dienst der Spitzenverbände der Krankenkassen e. V., Essen

4. Medizinischer Dienst der Spitzenverbände der Krankenkassen e. V. (1999) Positionspapier zu Rolle und Funktion des Medizinischen Dienstes im Verhältnis zu seinen Trägern. Beschlussfassung im Arbeitskreis I am 17.12.1999. Medizinischer Dienst der Spitzenverbände der Krankenkassen e. V., Essen

5. Medizinischer Dienst der Spitzenverbände der Krankenkassen e.V., Arbeitsgruppe Ü 1-MedJur, M10-MedJur (2000) Leitfaden für die Zusammenarbeit zwischen Krankenkassen und MDK bei Erstattungsansprüchen zwischen Krankenkassen und Unfallversicherungsträgern (KV/UV), 1. Ausg., Stand Juli 2000. Medizinischer Dienst der Spitzenverbände der Krankenkassen e. V., Essen

6. Mittelstaedt G von (2005) Selbstverständnis und Rollenwahrnehmung des MDK-Gutachters. Hauptstadtkongress 2005 Medizin und Gesundheit: Der MDK im Spannungsfeld zwischen Kontrolle und Dienstleistung. Berlin, 15.-17.06.2005 